\title{
Katherine Philips traductora de Saint-Amant y Corneille
}

Fecha de recepción: 23 de febrero de 2007

Ángeles García CALDERón

Universidad de Córdoba

Fecha de aceptación: 4 de mayo de 2007

Resumen: Trabajo que trata sobre una de las primeras mujeres autoras inglesa de renombre, Katherine Philips, conocida en su círculo literario como "la incomparable Orinda", que, además de su faceta de poeta llegó a gozar de fama literaria como traductora de dos escritores franceses: el poeta barroco Saint-Amant y el dramaturgo creador de la denominada tragedia psicológica en Francia: Pierre Corneille.

Palabras clave: Poesía inglesa, Feminismo, Traducción.

Abstract: In this article I approach the work of one of the most prominent English women writers, Katherine Philips, known in her literary coterie as the "uncomparable Orinda" ("The Matchless Orinda"). She was not only well reputed as a poet, but also she gained success as a translator of two French writers: the baroque poet SaintAmant and the playwright responsible for the creation of the so called psychological tragedy in France, Pierre Corneille.

Key words: English poetry, Feminism, Translation.

\section{INTRODUCCIÓN: LA AUTORA}

Nacida con el comienzo del año 1932 en el seno de la familia Fowler, su padre era un comerciante londinense de mentalidad moderadamente puritana. La niña fue escolarizada en la prestigiosa "Mrs. Salmon's School" de la localidad de Hackney (Londres), conociendo allí entre otras a Mary Autrey, que encarnaría el papel de 'Rosania' de su poema del mismo nombre; también se relacionó allí con Mary Harvey, que encarnó el papel de 'Silvander'. Su padre moriría en 1639, pero su madre volvió a contraer nupcias en 1646 con un barón de Gales. Éste casó a Katherine con un tal James Philips, pariente suyo, cuando aún era una jovencita de 16 años y él un hombre maduro de 52 años, parlamentario notable que respaldaba la causa puritana y que firmaría la condena a muerte del rey Charles I en 1649. Parece que Katherine había comenzado a escribir poesía poco después de su matrimonio, hecho que no pasó desapercibido para el notable poeta metafísico Henry Vaughan, quien alabó los poemas de la ya denominada por esa época "The Matchless Orinda" en su obra Olor Iscanus. Posteriormente Vaughan publicó un poema que Catherine había escrito en memoria del poeta y dramaturgo William Cartwright. Desde ese momento comenzó a utilizar como pseudónimo el nombre de 'Orinda'. Tras 
el matrimonio de Mary Aubrey, con quien mantuvo correspondencia poética, su sustituta fue Anne Owen, a quien haría famosa como 'Lucasia'.

Durante la guerra civil Katherine albergó simpatías realistas a pesar de su educación puritana y de la influencia de su marido. De ahí que celebrara con júbilo la restauración monárquica de Carlos II en 1660, si bien no pudo evitar quedar comprometida en los asuntos de su marido. Por suerte, éste no sufrió pena alguna por su papel en la ejecución real, aunque perdió su posición de parlamentario y sus antiguas posesiones, debidas al agradecimiento de Cromwell, hubieron de ser restituidas a la corona. En última instancia a Katherine le sonrió la fortuna porque su marido gozó de consideración por parte del Maestro de Ceremonias de Charles II, Cotterell, quien interesado en conseguir la mano de Anne Owen con ayuda de Katherine, le ayudaría en pago intercediendo por su marido. A pesar de que Anne Owen no llegó a casarse con Cotterell, Katherine continuó su amistad con éste influyente personaje de la corte. De hecho, fue él quien preparó sus poemas para su publicación tras su prematura muerte de viruelas en 1664, poco después de que volviera de Irlanda de visitar a su amiga Anne.

Durante su vida Philips pudo ver dos de sus libros impresos: una traducción de la obra de Corneille La Mort de Pompée, que su amigo dramaturgo el conde de Orrery había representado en Dublín, ea impreso poco después en 1663; el segundo, no autorizado, fue Poems by the Incomparable Mrs K.P. publicado en 1664, retirado al poco ante las objeciones puestas por la propia Katherine. La edición autorizada de Cotterell de Poems vió la luz en 1667, con reediciones en 1669, 1678 y 1710 , lo que significó un gran éxito tratándose de una poetisa ya difunta. Sus cartas a 'Poliarco' (Cotterell) se publicaron en 1705. En su época Katherine Philips fue considerada una especie de contrapartida respetable a la figura descollante de Aphra Behn, considerada por no pocos destacados críticos como inmoral y vulgar. La poesía de 'Orinda' (Katherine) es refinada, por lo que en su tiempo fue muy celebrada como poetisa, ganando el aprecio de Dryden entre otros autores famosos. Los críticos feministas actuales ensalzan a 'Orinda' debido a su alabanza de la amistad platónica femenina y por el interés despertado entre los académicos por los círculos femeninos en el siglo XVII, aunque ha sido denostada por no pocos por no atreverse a ir más allá en su amistad del simple plano intelectual. Su traducción de Corneille es precisa y bien elaborada. Su poesía es elegante, pero en muchos pasajes es bastante vaga: está escrita en un estilo llano y directo, y a veces exhibe fuertes sentimientos, pero casi nunca trasciende lo convencional, cuando, tanto por su cultura como por su libertad de pensamiento y su posición social, podía haber escrito una poesía más honda y auténtica para la que parecía estar bien dotada. 


\section{LA SOLITUDE DE SAINT-AMANT}

Curiosa personalidad la de este escritor de familia de burgueses hugonotes cuyo padre navegó bastantes años como agente marítimo de la reina de Inglaterra, ocupando posteriormente una posición importante en el comercio de Rouen; ello le posibilitó al joven Antoine Girard la ocasión de tomar parte en expediciones marítimas que lo llevaron desde las costas occidentales de África hasta América, antes de recalar en París, hacia 1619. Su obra denota una vasta sensibilidad y cultura en literatura moderna, pero él mismo confiesa ignorar el latín y el griego; en París entra al servicio de Henri de Gondi, duque de Retz, al que acompaña a su feudo y a Belle-Isle (donde escribirá su primera composición poética: La Solitude); sus cualidades mundanas de buen conversador, bebedor, fumador $y$ gastrónomo lo introducen en la casa del conde de Harcourt al que acompaña en 1637 a España, Italia e Inglaterra. En 1654 se convierte en gentilhombre de la reina de Polonia, María de Gonzaga, volviendo de ese país por Suecia, donde conoce a la reina Cristina.

Sus aficiones le proporcionarán una injusta fama de 'goinfre' (glotón) que no se ajusta a la realidad: al mismo tiempo que frecuenta tabernas, mesones y restaurantes, asiste a lugares como el Hotel de Liancourt, el Hotel de Mme de Rambouillet y las sesiones de la inaugurada Academia Francesa (1635), de la que es uno de sus miembros fundadores. Tras su vuelta de Polonia su inquietud viajera declina; las críticas de Boileau a su obra hacen que esta se vea menospreciada o tratada de un modo muy severo, constatando únicamente el mal gusto de algunas piezas escritas a la manera de Marino. Reivindicada su figura por Théophile Gautier (Les Grotesques) en 1844, aún hoy sigue atribuyéndosele fama de bohemio, juerguista y glotón.

En cuanto al poema, la Solitude de Saint-Amant puede ser datada en 1617. Es muy probable que tanto Saint-Amant haya tomado el título de la literatura española, ya que su Solitude es exactamente una meditación a la manera del gran poema del cordobés Góngora, quien concibe su obra en torno al eje central del 'solitario', tema esencial del barroco, que cultivan en la Península Ibérica sobre todo dos autores: Pedro de Espinosa y la portuguesa Bernarda Ferreira de la Cerda ${ }^{1}$. Veamos el poema del autor francés y la traducción de Katherine Philips:

\footnotetext{
${ }^{1}$ Para esta cuestión remitimos al artículo de Miguel Á. GARCíA: "Las Solitudes en la poesía francesa de principios del XVII: Théophile de Viau y Saint-Amant", Traducción, Cultura e Inmigración. Reflexiones interdisciplinares, Granada, Editorial Atrio, 2004, pp. 295-317.
} 


\section{Traducción de Katherine Philips de $L a$ Solitude de Saint-Amant}

O! Solitude, my sweetest choice

Places devoted to the night,

Remote from tumult, and from noise,

How you my restless thoughts delight!

O Heavens! what content is mine,

To see those trees which have appear'd

From the nativity of Time,

And which hall ages have rever'd,

To look to-day as fresh and green,

As when their beauties first were seen!

A cheerful wind does court them so, And with such amorous breath enfold, That we by nothing else can know, But by their height that they are old. Hither the demi-gods did fly

To seek the sanctuary, when

Displeased Jove once pierc'd the sky,

To pour a deluge upon men,

And on these boughs themselves did save,

When they could hardly see a wave.

Sad Philomel upon this thorn

So curiously by Flora dress'd,

In melting notes, her case forlorn,

To entertain me, hath confess'd.

$\mathrm{O}$ ! how agreeable a sight

These hanging mountains do appear,

Which the unhappy would invite

To finish all their sorrows here,

When their hard fate makes them endure

Such woes, as only death can cure.

What pretty desolations make

These torrents vagabond and fierce,

Who in vast leaps their springs forsake,

This solitary Vale to pierce.

Then sliding just as serpents do

Under the foot of every tree,

Themselves are changed to rivers too,

Wherein some stately Nayade,

\section{Antoine Girard de Saint-Amant: $L a$ Solitude A Alcidon ${ }^{2}$}

O que j'ayme la solitude!

Que ces lieux sacrez à la nuit, Esloignez du monde et du bruit, Plaisent à mon inquietude!

Mon Dieu! que mes yeux sont contens

De voir ces bois, qui se trouverent

A la nativité du temps,

Et que tous les siècles reverent,

Estre encore aussi beaux et vers,

Qu'aux premiers jours de l'univers!

Un gay zephire les caresse

D'un mouvement doux et flatteur.

Rien que leur extresme hauteur

$\mathrm{Ne}$ fait remarquer leur vieillesse. Jadis Pan et ses demy-dieux

$Y$ vindrent chercher du refuge,

Quand Jupiter ouvrit les cieux

Pour nous enoyer le deluge,

Et, se sauvans sur leurs rameaux,

A peine virent-ils les eaux.

Que sur cette espine fleurie

Dont le printemps est amoureux,

Philomele, au chant langoureux,

Entretient bien ma resverie!

Que je prens de plaisir à voir

Ces monts pendans en precipices,

Qui, pour les coups du desespoir,

Sont aux malheureux si propices,

Quand la cruauté de leur sort,

Les force a rechercher la mort!

Que je trouve doux le ravage

De ces fiers torrens vagabonds,

Que se precipitent par bonds

Dans ce vallon vert et sauvage!

Puis, glissant sour les arbrisseaux,

Ainsi que des serpens sur l'herbe,

Se changent en plaisans ruisseaux,

Où quelque Naïade superbe

${ }^{2}$ Referencia a Charles Maignart de Bernières (1616-1662), Presidente del Parlamento de Rouen. 
As in her native bed, is grown

A queen upon a crystal throne.

This fen beset with river-plants, O! how it does my sense charm! Nor elders, reeds, nor willows want, Which the sharp steel did never harm. Here Nymphs which come to take the air, May with such distaffs furnish'd be,

As flags and rushes can prepare Where we the nimble frogs may see, Who frighted to retreat do fly If an approaching man they spy.

Here water-fowls repose enjoy, Without the interrupting care, Lest Fortune should their bliss destroy By the malicious fowler's snare. Some ravish'd with so bright a day, Their feathers finely prune and deck; Others their amorous heats allay, Which yet the waters could not check: All take their innocent content In this their lovely element.

Summer's, nor Winter's bold approach, This stream did never entertain; Nor ever felt a boat or coach, Whilst either season did remain. No thirsty traveller came near, And rudely made his hand his cup; Nor any hunted hind hath here Her hopeless life resigned up; Nor ever did the treacherous hook Intrude to empty any brook.

What beauty is there in the sight Of these old ruin'd castle-walls Of which the utmost rage and spight Of Time's worst insurrection falls? The witches keep their Sabbath here, And wanton devils make retreat. Who in malicious sport appear, Our sense both to afflict and cheat; And here within a thousand holes Are nest of adders and of owls.
Regne comme en son lict natal, Dessus un throsne de christal!

Que j'ayme ce marets paisible! Il est tout bordé d'aliziers, D'aulnes, de saules et d'oziers, A qui le fer n'est point nuisible. Les nymphes, y cherchans le frais, S'y viennet fournir de quenouilles, De pipeaux, de joncs et de glais;

Où l'on voit sauter les grenouilles,

Qui de frayeur s'y vont cacher

Si tost qu'on veut s'en approcher.

Là, cent mille oyseaux aquatiques Vivent, sans craindre, en leur repos, Le giboyeur fin et dispos,

Avec ses mortelles pratiques.

L'un tout joyeux d'un si beau jour,

S'amuse à becqueter sa plume;

L'autre allentit le feu d'amour

Qui dans l'eau mesme se consume,

Et prennent tous innocemment

Leur plaisir en cet element.

Jamais l'esté ny la froidure N'ont veu passer dessus cette eau Nulle charrette ny batteau, Depuis que l'un et l'autre dure; Jamais voyageur alteré N'y fit servir sa main de tasse; Jamais chevreuil desesperé N'y finit sa vie à la chasse; Et jamais le traistre hameçon N'en fit sortir aucun poisson.

Que j'ayme à voir la décadence

De ces vieux chasteaux ruinez,

Contre qui les ans mutinez

Ont deployé leur insolence!

Les sorciers y font leur sabat;

Les demons follets s'y retirent,

Qui d'un malicieux ébat

Trompent nos sens et nous martirent;

Là se nichent en mille troux

Les couleuvres et les hyboux. 
The raven with his dismal cries, That mortal augury of Fate, Those ghastly goblins ratifies, Which in these gloomy places wait. On a curs'd tree the wind does move A carcase which did once belong To one that hang'd himself for love Of a fair Nymph that did him wrong, Who thought she saw his love and truth, With one look would not save the youth.

But Heaven which judges equally, And its own laws will still maintain, Rewarded soon her cruelty With a deserv'd and mighty pain: About this squalid heap of bones, Her wand'ring and condemned shade, Laments in long and piercing groans The destiny her rigour made, And the more to augment her right, Her crime is ever in her sight.

There upon antique marbles trac'd, Devices of past times we see, Here age ath almost quite defac'd, What lovers carv'd on every tree. The cellar, here, the highest room Receives when its old rafters fail, Soil'd with the venom and the foam Of the spider and the snail:

And th'ivy in the chimney we

Find shaded by a walnut tree.

Below there does a cave extend, Wherein there is so dark a grot, That should the Sun himself descend, I think he could not see a jot. Here sleep within a heavy lid In quiet sadness locks up sense, And every care he does forbid, Whilst in arms of negligence, Lazily on his back he's spread, And sheaves of poppy are his bed.

Within this cool and hollow cave, Where Love itself might turn to ice, Poor Echo ceases not to rave
L'orfraye, avec ses cris funebres, Mortels augures des destins,

Fait rire et dancer les lutins

Dans ces lieux remplis de tenebres.

Sous un chevron de bois maudit Y branle le squelette horrible D'un pauvre amant qui se pendit Pour une bergère insensible, Qui d'un seul regard de pitié Ne daigna voir son amitié.

Aussi le Ciel, juge équitable, Qui maintient les loix en vigueur, Prononça contre sa rigueur Une sentence epouvantable: Autour de ces vieux ossemens Son ombre, aux peines condamnée, Lamente en longs gemissemens Sa malheureuse destinée, Ayant, pour croistre son effroy, Tousjours son crime devant soy.

Là se trouvent sur quelques marbres Des devises du temps passé; Icy l'âge a presque effacé

Des chiffres taillez sur les arbres; Le plancher du lieu le plus haut Est tombé jusques dans la cave, Que la limace et le crapaud Souillent de venin et de bave; Le lierre y croist au foyer, A l'ombrage d'un grand noyer.

Là dessous s'estend une voûte Si sombre en un certain endroit, Que, quand Phebus y descendroit, Je pense qu'il n'y verroit goutte; Le Sommeil aux pesans sourcis, Enchanté d'un morne silence, $Y$ dort, bien loing de tous soucis, Dans les bras de la Nonchalence, Laschement couché sur le dos Dessus des gerbes de pavots.

Au creux de cette grotte fresche, Où l'Amour se pourroit geler, Echo ne cesse de brusler 
On her Narcissus wild and nice:

Hither I softly steal a thought,

And by the softer music made

With a sweet lute in charms well taught,

Sometimes I flatter her sad shade,

Whilst of my chords I make such choice,

They serve as body to her voice.

When from these ruins I retire,

This horrid rock I do invade,

Whose lofty brow seems to inquire

Of what materials mists are made:

From thence descending leisurely

Under the brow of this steep hill

It with great pleasure I descry

By waters undermin'd, until

They to Palaemon's seat did climb,

Compos'd of sponges and of slime.

How highly is the fancy pleas'd

To be upon the Ocean's shore,

When she begins to be appeas'd

And her fierce billows cease to roar

And when the hairy Tritons are

Riding upon the shaken wave,

With what strange sounds they strike the air

Of their trumpets hoarse and brave,

Whose shrill reports does every wind

Unto his due submission bind!

Sometimes the sea dispels the sand, Trembling and murmuring in the bay,

And rolls itself upon the shells

Which it both brings and takes away.

Sometimes exposed on the strand,

Th'effect of Neptune's rage and scorn,

Drown'd men, dead monsters cast on land,

And ships that were in tempests torn,

With diamonds and ambergreece,

And many more such things as these.

Sometimes so sweetly she does smile,

A floating mirror she might be,

And you would fancy all that while

New Heavens in her face to see:

The Sun himself is drawn so well,

When there he would his picture view,
Pour son amant froid et revesche, Je m'y coule sans faire bruit, Et par la celeste harmonie

D'un doux lut, aux charmes instruit, Je flatte sa triste manie

Faisant, repeter mes accords

A la voix qui luy sert de corps.

Tantost, sortant de ces ruines, Je monte au haut de ce rocher, Dont le sommet semble chercher En quel lieu se font les bruïnes; Puis je descends tout à loisir, Sous une falaise escarpée, D'où je regarde avec plaisir L'onde qui l'a presque sappée Jusqu'au siege de Palemon, Fait d'esponges et de limon.

Que c'est une chose agreable D'estre sur le borde de la mer, Quand elle vient à se calmer Après quelque orage effroyable! Et que les chevelus Tritons, Hauts, sur les vagues secouées, Frapent les airs d'estranges tons Avec leurs trompes enrouées, Dont l'eclat rend respectueux Les ventes les plus impetueux.

Tantost l'onde broüillant l'arène, Murmure et fremit de courroux Se roullant dessus les cailloux Qu'elle apporte et qu'elle r'entraine. Tantost, elle estale en ses bords,

Que l'ire de neptune outrage,

Des gens noyex, des monstres morts,

Des vaisseaux brisez du naufrage,

Des diamans, de l'ambre gris,

Et mille autres choses de pris.

Tantost, la lus claire du monde, Elle semble un miroir flottant, Et nous represente à l'instant Encore d'autres cieux sous l'onde. Le soleil s'y fait si bien voir, $Y$ contemplant son beau visage, 
That our eye can hardly tell Which is the false Sun, which the true; And lest we give our sense the lie, We think he's fallen from the sky.

Bernieres! for whose beloved sake My thoughts are at a noble strife, This my fantastic landskip take, Which I have copied from the life. I only seek the deserts rough, Where all alone I love to walk, And with discourse refin'd enough, My Genius and the Muses talk; But the converse most truly mine, Is the dear memory of thine.

Thou mayst in this Poem find, So full of liberty and heat,

What illustrious rays have shin'd To enlighten my conceit:

Sometimes pensive, sometimes gay, Just as that fury does control, And as the object I survey

The notions grow up in my soul, And are as unconcern'd and free As the flame which transported me.

O! how I Solitude adore,

That element of noblest wit, Where I have learnt Apollo's lore, Without the pains to study it: For thy sake I in love am grown With what thy fancy does pursue; But when I think upon my own, I hate it for that reason too.

Because it needs must hinder me From seeing, and from serving thee.
Qu'on est quelque temps à savoir Si c'est luy-mesme, ou son image, Et d'abord il semble à nos yeux

Qu'il s'est laissé tomber des cieux.

Bernières, pour qui je me vante De ne rien faire que de beau, Reçoy ce fantasque tableau Fait d'une peinture vivante, Je ne cherche que les deserts, Où, resvant tout seul, je m'amuse A des discours assez diserts De mon genie avec la muse; Mais mon plus aymable entretien C'est le ressouvenir du tien.

Tu vois dans cette poësie Pleine de licence et d'ardeur Les beaux rayons de la splendeur Qui m'esclaire la fantaisie: Tantost chagrin, tantost joyeux Selon que la fureur m'enflame, Et que l'objet s'offre à mes yeux, Les propose me naissent en l'ame, Sans contraindre la liberté

Du demon qui m'a transporté.

O que j'ayme la solitude! C'est l'element des bons esprits, C'est par elle que j'ay compris L'art d'Apollon sans nulle estude. Je l'ayme pour l'amour de toy, Connaissant que ton humeur l'ayme Mais quand je pense bien à moy, Je la hay pour la raison mesme Car elle pourroit me ravir L'heur de te voir et te servir

Por lo que concierne a la traducción de K. Philips, está realizada en octosílabos, del mismo modo que el original; la traductora huye del literalismo fácil, asimilando las ideas que expone el autor francés y tamizándolas por medio de su propia sensibilidad creativa; así, la frase exclamativa del $1^{\text {er }}$ verso del original se convierte en reflexiva en la traducción, elaborándolas más en otras ocasiones como en el verso 4: "mon inquietude" = "you my restless thoughts delight!". Este recurso se repite con profusión con el fin de adaptar la frase precisa del francés, de 
naturaleza polisilábica, a la morfología del verbo inglés, tendente al monosilabismo. No obstante, hay varios galicismos en la obra traducida porque las necesarias palabras polisilábicas son más precisas en la descripción de sentimientos: "solitude" (en lugar de "loneliness"), "nativity" (birth"), "deluge", "sanctuary", "vagavond", etc. Veamos otros ejemplos de cambio sintáctico, de cambio, 'modulativo' (noción de equivalencia de Vinay y Darbelnet), y de cambio semántico: traducción:

En la estrofa tercera el discurso francés es más expositivo que la

"Que sur cette espine fleurie

Dont le printemps est amoureux,

Philomele, au chant langoureux,

Entretient bien ma resverie!..."

"Sad Philomel upon this thorn,

So curiously by Flora dress'd,

In melting notes, her case forlorn,

To entertain me, hath confess'd..."

En la cuarta estrofa hallamos otro ejemplo de cambio 'modulativo':

"Que je trouve doux le ravage

De ces fiers torrens vagabonds,..."

En inglés, Philips prefiere la expresión impersonal:

"What pretty desolations make

These torrents vagabond and fierce,..."

En los últimos versos de la misma estrofa, la idea que transmite el texto inglés no es la del original, pues se utiliza la noción de reina, no la del verbo reinar:

"As in her native bed, is grown
A queen upon a crystal throne."
"Regne comme en son lict natal,
Dessus un throsne de christal!"

De un modo general la traducción, que sigue más que la linealidad del verso las proposiciones expuestas, se aparta del original, sobre todo en la sintaxis por la transposición de elementos del discurso.

En cuanto al poema original, éste gozó de un gran predicamento en Inglaterra quizá debido a los viajes del autor a ese país y disfrutó de una 
influencia duradera en la poesía inglesa. Además de la traducción al inglés (que podemos encontrar en su obra Poems, publicada póstumamente en 1667), una adaptación de "La Solitude" aparece en 1716 en la edición completa de la antología de Dryden y Tompson; posteriormente en 1780, en la antología de poemas escogidos (volumen I) publicada por Nichols ${ }^{3}$. El éxito prolongado del poema se explica debido a su tonalidad moderna: en él se encuentran resumidas las múltiples facetas de la sensibilidad prerromántica inherente a la poesía inglesa nocturna de la melancolía en el $\mathrm{XVIII}$, de la que Saint-Amant es considerado como un precursor por este poema concreto en el que el escritor apela a la sensibilidad más que a la inteligencia, apareciendo el paisaje como el reflejo de un estado del alma. En este aspecto, Saint-Amant es un adelantado a su tiempo, ya que la sensibilidad romántica inspira su obra: siempre atento a los espectáculos alterables e irregulares de la Naturaleza, los traduce y expresa con cualidades reales de fuerza y pintoresquismo. Escrita en Belle-Isle, donde permaneció largo tiempo, su "Solitude" deja volar la imaginación, ensoñando meditaciones ante las olas o siguiendo el vuelo de las gaviotas que se elevan y descienden trazando extrañas y fugaces figuras ante el sol deslumbrador, cual si de un espejismo se tratara. Diríase que el poeta ama la soledad porque parece corresponderse con su humor sombrío y lo mantiene en una tristeza agradable de la que no quiere apartarse; por eso, busca la sombra y la noche que están en armonía con su espíritu inquieto y adulan su tormento. Se pasea por los bosques, escucha el canto lánguido del ruiseñor y expresa su admiración por los aspectos más agrestes y pintorescos de la naturaleza: montañas, torrentes, valles solitarios; describe luego las ruinas de un viejo castillo, abandonado a las culebras y las lechuzas, frecuentado por las brujas y los demonios. Observa con espanto el esqueleto de un ahorcado, evocando de paso la historia de este hombre que se suicidó por el amor de una cruel mujer cuyo fantasma, obsesionado por los remordimientos, vuelve a vagar y purgar su pena en estos lugares. Después, el poeta se refugia en una gruta oscura y allí el sueño calma sus angustias; prosigue su paseo cerca del océano, expresando su sensibilidad por la quietud y dulzura de un paisaje marítimo y por el chapoteo del agua, que lo incita al ensueño y a la meditación. Todo ello denota un gusto muy original por la descripción, que se percibe en la vivacidad de las impresiones y la plenitud de sensaciones y sentimientos.

\footnotetext{
${ }^{3}$ John Nichols: A Selected Collection of Miscellaneous Poems with Historical and Biographical Notes, 8 vols., London, 1780-1782 (New York, Kraus Reprint Society, 1969).
} 


\section{LA MORT DE POMPEE DE CORNEILLE}

El gran éxito de Pierre Corneille en Francia va a verse reflejado también en Inglaterra, donde tanto sus temas como sus concepciones de la dramaturgia y su creación de la tragedia psicológica con Le Cid gozarán de gran predicamento entre los dramaturgos ingleses. Antes de tratar de la influencia de Corneille en Inglaterra, parece lógico partir de la definición de Aristóteles, a la cual se refieren constantemente teóricos y poetas de su tiempo en Francia:

La tragédie est l'imitation de quelque action sérieuse complète, ayant une certaine étendue, par un discours orné, dont les ornements ne se trouvent pas tous dans chaque partie, sous forme narrative et non pas narrative, employant la terreur et la pitié pour purger les passions de ce genre ${ }^{4}$.

En el momento en que el público francés comienza verdaderamente a interesarse por el teatro serio, ya en los últimos años del siglo XVI, pone sus ojos en la tragicomedia y en la pastoral dramática, que influyen en poner de moda la tragedia, consagrada a la representación de acciones violentas y desmedidas, con un lenguaje brillante y una fantasía que no retrocede ante la inverosimilitud. Jean de Schelandre y Alexandre Hardy renovarán las obras, representadas en el Hôtel de Bourgogne para un público ruidoso pero interesado. La nueva generación de autores dramáticos que aparece en la década de $1630^{5}$, a la vez que perfeccionan su concepción de la tragedia, tendrán en cuenta los argumentos utilizados por los teóricos para conducir al teatro a la "mesure" y a la "raison".

Para que la tragedia adquiera un estatus propio en Francia se dan una conjunción de factores que propiciarán la dignificación y el salto adelante necesario para las representaciones "serias", y que se pueden resumir en tres:

$\left.1^{a}\right)$ La intensa actividad teórica que va unida al descubrimiento de la Poética de Aristóteles, la cual nace en Italia con los tratadistas desde 1560 y experimentará una gran efervescencia en Francia alrededor de 1620 con los nombres de Piccolomini, Castelvetro, Escalígero, etc. Del prodigioso conjunto de teóricos y críticos que componen el Humanismo italiano, las obras de tres de ellos sobresalen: De Arte poetica de Vida (1527), Poetices libri septem de Escalígero (1561) y Poétique d'Aristote de Castelvetro

\footnotetext{
${ }^{4}$ La traducción castellana de la edición trilingüe de Valentín García Yebra es la siguiente: "Es, pues, la tragedia imitación de una acción esforzada y completa, de cierta amplitud, en lenguaje sazonado, separada cada una de las especies [de aderezos] en las distintas partes, actuando los personajes y no mediante relato, y que mediante compasión y temor lleva a cabo la purgación de tales afecciones" (Madrid, Gredos, p. 145).

5 Fundamentalmente Georges de Scudéry (1601-1667), Jean de Mairet (1604-1686), Jean Rotrou (1609-1650) y Pierre Corneille (1606-1684).
} 
(1570). Todos ellos son leídos, estudiados y analizados como intermediarios del pensamiento de Aristóteles, siendo cuatro escritores franceses los que debatirán las reglas de la tragedia, imponiendo definiciones rigurosas sobre la "vraisemblance" y la "bienséance", que ayudarán a la separación de los géneros teatrales y matizarán la exigencia de las tres unidades: Chapelain, La Ménardiere, Sarasin y el "abbé" d'Aubignac. Citemos las aportaciones esenciales de ellos, así como las anteriores cronológicamente:

1549: Deffence et Illustration de la langue françoise de Du Bellay

1555: Art poétique de Peletier du Mans

1561: Poetices libri septem de Escalígero

1570: la Poetica d'Aristote vulgarizata de Castelvetro

1572: De l'art de la tragédie de Jean de La Taille

1605: Art poétique de Vauquelin de La Fresnaye (iniciado en 1574)

1611: De tragœediae constitutione liber del holandés Daniel Heinsius

1630: Lettre à Godeau sur les vingt-quatre heures de Chapelain

1631: "Préface" de La Silvanire de Mairet

1639: Apologie du théâtre de Scudéry

Discours de la tragédie de Sarasin

Poétique de La Ménardière

1647: Institutiones Poeticae de Gerhard Johann Vossius

1657: La Pratique du théâtre del "abbé" d'Aubignac (comenzada en 1640)

1660: Discours sur le poème dramatique de Corneille

$2^{a}$ ) El esfuerzo de codificación, animado constantemente por el poder (Richelieu jugará hasta un muerte un papel fundamental), que favorece el desarrollo del teatro, que propicia un nuevo público más culto y educado. Entre 1630 y 1660 -fecha de la publicación teórica de Pierre Corneille-, el prestigio de la tragedia va a crecer sin parar: si de 1630 a 1639 se habían representado en París 38 tragedias por 80 tragicomedias, el número de unas y otras será prácticamente similar de 1640 a 1659.

$3^{\text {a) }}$ El constante progreso de las técnicas teatrales y la aparición de una línea ininterrumpida de grandes actores ${ }^{6}$, que dan con sus representaciones el tono adecuado que necesitaba la tragedia y que hasta entonces no había tenido.

El hecho de que el más imitado de los autores franceses sea Pierre Corneille se debe a las innovaciones y concepción que tiene el autor normando de la representación teatral. Corneille piensa que la naturaleza seria de la acción y la dignidad de los personajes bastan para diferenciar la

${ }^{6}$ Bellerose, Floridor, Montfleury, la Villiers, la Du Parc, la Champmeslé, etc. 
tragedia de la comedia. En lo referente a la acción, todos los teóricos están de acuerdo: debe provenir de la historia, sobre todo de la griega y romana; de hecho, los temas modernos son cada vez más rechazados por el público. Pregunta importante era si la tragedia debía contener muchos acontecimientos o no. Jean Chapelain ${ }^{7}$ explica en 1630 que la tragedia debe ser, en este punto, extremadamente "dépouillée":

Les anciens ont avec grand jugement réservé le théâtre à la catastrophe seulement, comme à celle qui contenait en vertu toute la force des choses qui la précédaient... le poème dramatique ne doit contenir qu'une action et encore de bien médiocre longueur... ; d'autre sorte, elle embarrasserait la scène et surchargerait extrêmement la mémoire ${ }^{8}$.

A este respecto, los textos esenciales para un mejor conocimiento de las teorías dramáticas de la época son La Pratique du théâtre ${ }^{9}$ del abbé d'Aubignac (1657) y los tres Discours que Corneille sitúa a modo de prólogo en los tres volúmenes de sus obras, en 1660. D'Aubignac no piensa que haya que inventar una definición para la tragedia, que ya se encuentra en los autores griegos, se trata de seguir el desarrollo y las acciones de un destino heroico, es decir la imitación de las vidas y de los discursos heroicos. Más concretamente, lo que define su concepción de la tragedia es la importancia que concede a la acción, una acción que se basa en el movimiento de las pasiones.

Para defender su concepción de la tragedia d'Aubignac cita frecuentemente a Corneille, celoso de su libertad como autor y a pesar de los elogios del "abbé", expresó su desacuerdo al teorizar sobre la tragedia. El meollo de la cuestión para él está en que lo verosímil admite como materia de lo trágico lo necesario; dicho de otro modo, lo que no depende de nuestro gusto, sino que se impone al existir o haber existido, estando avalado por la autoridad de la historia. Así, según él, basándose en el propio Aristóteles, el poeta debe representar las cosas como han sido, o como dicen que han sido, o como han debido ser.

Las obras de los dramaturgos franceses tuvieron una influencia muy directa en la tragedia inglesa durante la "Caroline era" (1625-1642), la

\footnotetext{
7 Hijo de un notario, Chapelain (1595-1674) gozaba en su tiempo de una reputación inmejorable por sus grandes conocimientos culturales, aunque no produjera una gran obra. Era el faro literario de la corte y de Richelieu, que lo encargó de llevar a cabo la fundación de la "Académie Française".

${ }^{8}$ Citado por Réné Bray: La formation de la doctrine classique en France, Paris, Librairie Nizet, 1927, p. 312; reediciones 1945 (de donde citamos), 1957 y 1966.

${ }^{9}$ D'Aubignac había redactado su obra crítica en los últimos años del ministerio de Richelieu, pero la muerte de este le hizo caer en un profundo desánimo; la retomó años más tarde para publicarla en 1657.
} 
"English Civil War" (1642-1651), el "English Interregnum" (1649-1660) y el período de la Restauración (1660-1689), sobre todo las piezas de Pierre Corneille. Una versión de Le Cid, del dramaturgo y traductor Joseph Rutter, fue llevada a la escena al poco tiempo de su estreno en Francia y "before their Majesties at Court and on the Cockpitt Stage in Drury Lane". Se trata de la primera traducción de Corneille al inglés, que fue seguida en 1655 y 1656, por dos versiones no muy afortunadas en verso libre de Polyeucte y Horace, de Sir William Lower ${ }^{10}$.

Aclaremos que, antes de la traducción de Katherine Philips, en 1643, había habido otras adaptaciones de obras de teatro francesas ${ }^{11}$, entre las que se pueden destacar las siguientes:

- En 1594 Thomas Kid había traducido Cornélie, publicada en 1574 por el autor francés trágico más grande del Renacimiento Robert Garnier $^{12}$; en la obra evocaba la desesperación de la mujer de Escipión e hija de Catón.

- Christopher Marlowe traduce el libro primero de la Farsalia (De Bello Civili) de Lucano, impresa en 1600 bajo el título de Lucans First Book translated line for line by Chr. Marlon, traducción que comprende 694 versos.

- William Shakespeare se inspirará para su Anthony and Cleopatre (h. 1607-1608) en la traducción inglesa de Thomas North (1573) de las Vidas paralelas de Plutarco ${ }^{13}$.

- George Chapman editará su Caesar and Pompey (h. 1612-1613).

- John Fletcher escribe junto con Philips Massinger: The False One, influenciada por la obra de Shakespeare, representada en 1619 e impresa en 1647; trata de la historia de Julio César y Cleopatra, siendo su fuente La Farsalia de Lucano.

- Thomas May publica, en 1626, The Tragedy of Cleopatra, Queen of Egypt.

\footnotetext{
${ }_{11}^{10}$ Ninguna de las dos obras parece que se llevara a la escena.

Para este punto son recomendables los estudios siguientes: Alexander Maclaren Witherspoon: The Influence of Robert Garnier on Elizabethan Drama, New Haven, Yale University Press, 1924; Eugene M. Waith: "The Death of Pompey: English Style, French Style", Shakespeare and Dramatic Tradition, Edited by William R. Elton and William B. Long. Newark, University of Delaware Press, 1989, pp. 276-85; Jean-Claude Ternaux: Lucain et la littérature de l'âge baroque en France. Citation, imitation et création, Paris, Honoré Champion, 1998.

${ }^{12}$ Obra perteneciente a la trilogía sobre las guerras civiles romanas de Robert Garnier (15441590): Porcie (1568), Cornélie (1574) y Marc-Antoine (1578).

${ }^{13}$ Thomas North había llevado a cabo su traducción de la francesa de Jacques Amiot (1559).
} 
No obstante, las obras maestras del teatro francés eran prácticamente desconocidas, en lo que a traducciones se refiere, convirtiéndose en un gran éxito la traducción que en 1663 realizó Katherine Philips de La Mort de Pompée ${ }^{14}$ de Corneille. Su versión, en verso rimado, fue representada en Dublín a principios de 1663 y luego en Londres, incrementando su reputación ya considerable de poetisa y siendo publicada el mismo año ${ }^{15}$. Katherine Philips, animada por el éxito de su Pompey, comenzó a traducir otra obra de Corneille, Horace, pero murió antes de terminarla, siendo completada su traducción por el poeta irlandés John Denham, y publicada en 1669.

\section{CONCLUSION}

En un tiempo en el que la mujer reduce prácticamente sus labores a la casa y al cuidado de los hijos, ya se pueden encontrar figuras que se rebelan contra esta "asignación" de papeles a los sexos. En este terreno, la vida de Katherine Philips, a pesar de ser tan corta, es una auténtica reivindicación de lo que ella entiende por su libertad como ser humano, tanto en el terreno afectivo como en el "mental". En el primero exponiendo claramente sus ideas respecto a la relación humana al fundar "The Society of Friendships" (1651-1661, círculo semi-literario compuesto en un principio por mujeres pero en el que no se excluía a los hombres) a la vez que no sentía pudor alguno en ir más allá de los afectos normales en las relaciones entre miembros de un mismo sexo, aunque parece que todo lleva a pensar que se trataba de un amor platónico. En cuanto a su labor profesional como escritora, sus dotes quedan bien patentes en la espléndida expresión de sentimientos y afectos que podemos hallar en sus poemas, siendo asimismo notoria su labor como traductora, atreviéndose con autores tan "modernos" como Saint-Amant y tan importantes e influyentes en toda Europa como Pierre Corneille. Su labor en este último apartado merece ser destacada por encima de la de todos los traductores de su tiempo en Inglaterra: tanto en un autor como en otro, la comprensión de la obra está por encima de los virtuosismos o lucimientos personales, dando a conocer a los lectores de su época obras d los poetas y dramaturgos en boga.

El conjunto de su figura y su obra como poeta y traductora harán de Katherine Philips una de las escritoras más notables de su tiempo,

\footnotetext{
${ }^{14}$ Que, a pesar de ser de tema romano, estaba inspirada por la muerte de Richelieu el dos de diciembre de 1642, lo que se deduce fácilmente por los siguientes versos de la obra:

"Car c'est ne régner pas qu'être deux à régner,

Un roi qui s'y résout es mauvais politique". (vv. 232-233)

${ }^{15}$ En 1664 apareció otra versión de la obra de Corneille firmada "by certain Persons of Honour".
} 
posiblemente junto con Aphra Behn la más conocida de su siglo, sirviendo de modelo a seguir por las escritoras inglesas del siglo XVIII.

\section{REFERENCIAS BIBLIOGRÁFICAS}

a) Ediciones (totales o parciales de la obra de Katherine Philips)

Katherine PHILIPS, Pompey, 1663.

Katherine PHILIPS, Horace, conclusion by John Denham, 1664.

Katherine PHILIPS, Poems by the Incomparable Mrs. K.P., 1664.

Katherine PHILIPS, Poems by the most deservedly Admired Mrs. Katherine Philips, the Matchless Orinda, to which is added Monsieur Corneille's Pompey and Horace, Tragedies, 1667.

Katherine FOWLER PHILIPS, Poems by the Most Deservedly Admired Mrs. Katherine Philips, The Matchless Orinda, Introduction by Travis DuPriest, London, Herringman, 1667.

Katherine PHILIPS, Poems, 1678.

Katherine PHILIPS, Letters by the Late Celebrated Mrs. Katherine Philips (from Rochester's Familiar Letters), 1697 (1705).

Katherine PHILIPS, Letters From Orinda to Poliarchus, 1703.

Specimens of British Poetesses, ed. Alexander Dyce, London, T. Rodd, 1827.

Specimens of the British Poets, ed. Thomas Campbell, London, John Murray, 1844.

The Femle Poets of Great Britain, ed. Frederic Rowton, Philadelphia, Carey and Hart, 1849.

Minor Poets of the Caroline Period, ed. George Saintsbury, Oxford, Clarendon Press, 1905. (Considerada como la edición de referencia)

The Oxford Book of English Verse, ed. Arthur Quiller-Couch, Oxford, Clarendon Press, 1919.

The Oxford Book of Seventeenth Century Verse, eds. H. J. C. Grierson and G. Bullough, Oxford, Clarendon Press, 1934.

Elizabethan and Seventeenth-Century Lyrics, ed. Matthew W. Black, Philadelphia, J. B. Lippincott \& Co., 1938.

Poetry of the English Renaissance 1509-1660, eds. J. William Hebel and Hoyt H. Hudson, New York: F. S. Crofts \& Co., 1941.

Kissing the Rod: An Anthology of Seventeenth Century Women's Verse, ed. Germaine Greer, London, Virago, 1988.

The Collected Works of Katherine Philips, The Matchless Orinda. Volume I: Poetry, ed Patrick Thomas, Stump Cross, 1990.

New Oxford Book of Seventeenth Century Verse, ed. Alastair Fowler, 1991.

Collected Works of Katherine Philips edited by Elizabeth H. Hageman and Andrea Sununu, Oxford University Press, 1996. 
The Oxford Book of English Verse, ed. Christhopher Ricks, Oxford University Press, 1999.

Early Modern Women's Writing, ed. Paul Salzman, Oxford University Press, 2000.

British Poetry 1603-1660, eds. John P. Rumrich and Gregory Chaplin, W. W. Norton \& Company, New York-London, 2005.

b) Estudios

ADAM, Antoine: Théophile de Viau et la libre pensée française en 1620, Paris, Droz, 1935 (Genève, Slatkine Reprint, 1965).

ANDREADIS, Harriette: "The Sapphic-Platonics of Katherine Philips, 16321664", Signs: Journal of Women in Culture and Society 15, ํㅜ1, autumn 1989, pp. 34-60.

, "Re-configuring early modern friendship: Katherine Philips and homoerotic desire", Studies in English Literature, 1500-1900, 2006.

BORTON, S., Six modes of sensibility in Saint-Amant, The Hague, 1966.

BRASHEAR, Lucy, "The Forgotten Legacy of the 'Matchless Orinda'", AngloWelsh Review 65, 1979, pp. 68-76.

BuCKINGHAM, Elinor M.,"The Matchless Orinda", Sewanee Review 10, 1902, 269-284.

EZELL, Margaret J. M., "Reading pseudonyms in seventeenth-century English coterie literature", Essays in Literature, 1994.

FUKUI, Yoshio, Raffinement précieux dans la poésie française du XVII siècle, Paris, A. G. Nizet, 1964.

GourieR, F., Étude des œuvres poétiques de Saint-Amant, Genève-Paris, Droz-Minard, 1961.

HoBBY, Elaine, "Katherine Philips: Seventeenth Century Lesbian Poet", What Lesbians Do in Books, London, The Women's Press, 1992, pp. 183204.

KASTNER, L. E., "Saint-Amant and the english poets", Modern Language Review, 1931, págs. 180-182.

LAGNY, Jean: Le poète Saint-Amant (1594-1661). Essai sur sa Vie et ses CEuvres, Paris, A. G. Nizet, 1964.

Loscocco, Paula, "Manly Sweetness: Katherine Philips among the Neoclassicals", Huntington Library Quarterly 56, no 3, summer 1993, pp. 259-279.

LLEWELLYN, Mark, "Katherine Philips: Friendship, Poetry and Neo-Platonic Thought in Seventeenth Century England", Philological Quarterly, Vol. 81, 2002.

Moody, Ellen, "Orinda, Rosania, Lucasia et aliae: Towards a New Edition of the Works of Katherine Philips", Philological Quarterly, 66, 1987, pp. 325-354. 
PereuX, Guillaume, "Le Rendez-vous des Enfans sans souci". La poétique de Saint-Amant, Paris, Honoré Champion, 2002.

Revard, Stella P., "Katherine Philips, Aphra Behn, and the Female Pindaric", Representing Women in Renaissance England, edited by Claude J. Summers and Ted-Larry Pebworth, Columbia, University of Missouri Press, 1997, pp. 227-241.

ROBERT, William, Saint-Amant, Orinda and Dryden's Miscellany," English Language Notes, 1 (Sept- June 1963-64), pp. 191-96. , The Dating of Orinda's French translations, Philological Quarterly, 49, 1970, pp. 56-196.

SABATIER, Robert, "Saint-Amant le joyeux", Histoire de la poésie française. La Poésie du dix-septième siècle, Paris, Albin Michel, 1975.

SCODEL, Joshua, "The pleasures of restraint: the mean of coyness in Cavalier poetry", Criticism, 38, Spring 1996.

SOUERS, Philip Webster, The Matchless Orinda, Cambridge, Harvard University Press, 1931 (Johnson Reprints, 1969).

Stiebel, Arlene, "Not Since Sappho: The Erotic Poems of Katherine Philips and Aphra Behn", Homosexuality in Renaissance and Enlightenment England, ed. Claude J. Summers, Binghamton, New York, Haworth, 1992, pp. 153-171.

"Subersive Sexuality: Masking the Erotic in Poems by Katherine Philips and Aphra Behn" Renaissance Discources of Desire, eds. Claude J. Summers and Ted-Larry Pebworth, Columbia, University of Missouri Press, 1993, pp. 223-236.

SwAIM, Kathleen M., "Matching the 'Matchless Orinda' to Her Times", 16501850: Ideas, Aesthetics, and Inquiries in the Early Modern Era. Vol. 3, edited by Kevin L. Cope, New York, AMS Press, 1997, pp. 77-108.

THOMAS, Katherine Philips, University Of Wales Press, 1988.

VARENNE, Pierre, Le bon gros Saint-Amant, Rouen, 1917. 